\title{
PHENOMENOLOGY OF CRYSTALLINE BEAMS IN SMOOTH ACCELERATORS *
}

\author{
A.F. Haffmans, D. Maletić, A.G. Ruggiero, \\ Brookhaven National Laboratory, Upton, NY 11973 USA
}

\section{Abstract}

We present a phenomenology of crystalline beams in storage rings. We use the smooth approximation to solve the equations of a test particle moving in the focussing potential of the storage ring, and in that of the other ions. We find simple confinement, and stability conditions.

\section{GENERAL OVERVIEW}

When ions in a storage ring are cooled sufficiently, they undergo a phase transition to a crystalline ion beam. These crystalline beams have already been observed in Molecular Dynamics simulations and ion trap experiments [1], [2], [3]. Among other things, crystalline beams provide a new way to obtain intense ion beams. We present an analytical study of ground state crystalline beams. We examine the stability of the motion of a test particle in the crystalline beam in a storage ring consisting of linear elements. We find the beam's confinement and stability conditions. Using simple, arguments from classical dynamics, we obtain a succession of beam structures, as the ion denisty is increased.

We start with a collection of ions with mass number A, and atomic number $\mathrm{Q}$ already near zero-temperature equilibrium position. The crystalline beam consists of a bundle of substrings, with ions placed a distance $\lambda$ apart in the longitudinal direction. The space-charge force on a test particle in the beam is included as a perturbation in the familiar single particle equations of motion [4]. We perform a "Gedanken Experiment" in which we systematically increase the number of ions in the crystalline beam, and hence decrease $\lambda$. At each step we determine the stability of the equilibrium of the test particle. At a critical value $\lambda$, the focussing forces no longer balance the space charge and the orbits along which the substrings lie, undergo a pitchfork bifurcation [5]. Two new stable orbits with the same period originate out of the unstable orbit, according to the Poincaré-Bendixson theorem [6]. As the number of ions is increased further, the process repeats itself: each stable orbit will become usntable, and undergo a pitchfork bifurcation, doubling the number of substrings.

Therefore, the specifics of the ground state of the crystalline beam is a function of the ion denisty, and focussing properties of the storage ring. Each crystalline beam is characterized by a range of values of the inter-ion spacing $\lambda$. The upper limit is the ion spacing when the structure is formed, and the lower limit is the ion spacing when the structure becomes unstable, and undergoes a bifurcation. In this bifurcation picture, the lower limit of a structure corresponds roughly to the upper limit of the next structure.

\footnotetext{
*Work performed under the auspices of the U.S. D.O.E.
}

\section{EQUATIONS OF MOTION}

We use a curvilinear orthogonal coordinate system [7],[4], where $x$ and $y$ denote the horizontal and vertical direction, respectively, $\sigma$ the path length difference and $\delta$, the momentum error. The equations of motion of the test particle are derived from the quadratic Hamiltonian discussed in [7], viz.:

$$
\begin{aligned}
y^{\prime \prime}+K_{v}(s) y+\frac{Q r_{0}}{e A \beta^{2} \gamma^{3}} \frac{\partial \Phi_{s c}}{\partial y} & =0 \\
x^{\prime \prime}+K_{h}(s) x+\frac{Q r_{0}}{e A \beta^{2} \gamma^{3}} \frac{\partial \Phi_{s c}}{\partial x} & =h(s) \delta ; \\
\sigma^{\prime} & =\frac{\delta}{\gamma^{2}}+h x ; \\
\delta^{\prime} & =\frac{Q r_{0}}{e A \beta^{2} \gamma^{3}} \frac{\partial \Phi_{s c}}{\partial \sigma}
\end{aligned}
$$

The derivatives are with respect to the curvilinear longitudinal coordinate $s ; \beta$ and $\gamma$ are the usual relativistic factors, $h(s)$ is the curvature, and $K_{v, h}(s)$ the focussing functions which describe the storage ring. $\Phi_{s c}$ is the space-charge potential, and $r_{0}$ is the classical proton radius.

We expand the space charge potential $\Phi_{s c}$ to second order in small deviations around the (conjectured) equilibrium position of the test particle. After putting these expansions in the equations of motion, we obtain two sets of equations: The envelope equations determining the equilibrium position of the test particle in the storage ring, and those for the motion around this equilibrium.

For storage rings with smooth lattice functions and smooth bending we may use the smooth approximation. That is, we replace the local focussing forces $K_{h, v}(s)$ with their averages around the ring. The average value of the amplitude function is $\nu_{h, v} / R$. In addition, we replace the local curvature $h(s)$ by $1 / R$, i.e. its average around the ring. We will use this below to obtain estimates of the tune shift.

\section{CONFINEMENT}

Confinement in the transverse direction is provided by the focusing of the storage ring. The crystalline beam has to be closed, and have the same periodicity as the storage ring. We can use this closure, or isochonism condition $-\sigma^{\prime}=0-$ to aproximately integrate Eq. (3). We find, that the momentum error $\delta_{l}$ of the test particle varies linearly with its horizontal displacement from the (dilute beam) reference trajectory $x_{l}$. This approximation is appropriate only for a sufficiently smooth lattice [7]. Substituting our approximate expression for $\delta_{l}$ in Eq. (2) gives the envelope equations:

$$
x_{l}^{\prime \prime}+\left[K_{h}(s)-\frac{\gamma^{2}}{R^{2}}-\frac{4 \lambda_{c}^{3}}{g_{0} R^{2} \lambda^{3}} \mathcal{F}_{h}\right] x_{l}=0,
$$




$$
y_{l}^{\prime \prime}+\left[K_{v}(s)-\frac{4 \lambda_{c}^{3}}{g_{0} R^{2} \lambda^{3}} \mathcal{F}_{v}\right] y_{l}=0 .
$$

The kinematic term proportional to $\gamma^{2}$ is entirely due to the isochronism condition across one super-period. $\lambda_{c}$ is the characteristic lenght of the crystalline beam:

$$
\lambda_{c}=\left(\frac{Q^{2} r_{0} g_{0} R^{2}}{A \beta^{2} \gamma^{5}}\right)^{\frac{1}{3}},
$$

$\mathrm{R}$ is the radius of the storage ring, and $g_{0}$ is a numerical factor, approximately equal to $1.2 . \mathcal{F}_{h}$ and $\mathcal{F}_{v}$ are proportional to the first order derivative of the space charge potential around the equilibrium. These equations are identical to those of a test particle perturbed by a space charge force. Because the period of the crystalline beam has to be the same as that of the storage ring, the tune shift induced by the space charge should be large enough to reach the integral stopband [7], [4]. Using the smooth approximation, together with the familiar incoherent tune shift estimate to impose this condition, we obtain the horizontal and vertical confinement conditions:

$$
\frac{\lambda^{3}}{F_{h, v}}=\left(\lambda_{c} \overline{c_{h, v}}\right)^{3}
$$

with:

$$
\begin{aligned}
\overline{c_{h, v}} & =\left(\nu_{h, v} \overline{\delta_{h, v}}\right)^{-\frac{1}{3}}, \\
\overline{\delta \nu_{h}} & =\frac{1}{2}\left(\nu_{h}-m N_{p}\right)-\frac{\gamma^{2}}{2 \nu_{h}} \\
\delta \nu_{v} & =\frac{1}{2}\left(\nu_{v}-m N_{p}\right),
\end{aligned}
$$

$N_{p}$ is the super-period of the storage ring, and $m$ an integer. $\nu_{h, v}$ are the dilute beam tunes, and correspond to the working point of the storage ring. The term in $\gamma^{2}$ is subtracted from the actual distance of the horizontal working tune to the nearest stopband. Therefore, for the appearance of a horizontally extended "Crystalline Beam", the following condition needs to be satisfied:

$$
\gamma<\sqrt{2 \nu_{h} \delta \nu_{h}}
$$

This generalizes the condition obtained by Wei, Li and Sessler [3], and in particular implies that the storage ring has to be operated below the transition energy.

$\lambda$ is the same for all substrings, and we can combine the horizontal and vertical confinement condition into the envelope function W:

$$
W=\frac{\mathcal{F}_{h}}{\mathcal{F}_{v}}=\frac{\bar{c}_{v}^{3}}{\bar{c}_{h}^{3}}=1-\Delta \propto \frac{K_{h}}{K_{v}},
$$

where $\Delta$ is the defocussing. Furthermore, this implies that the solutions of the envelope equations are invariant under reflections, and rotations.

The confinement in the longitudinal direction is provided by the repulsion between the ions in the "Crystalline Beam". In the absence of curvature, there is no net longitudinal force when the ions are in their equilibrium position. The longitudinal force between the different strings in the crystalline beam is zero, when the logitudinal shift between them is either either 0 or $\lambda / 2$. Right after a bifurcation, some of the substrings have a relative shift of $\lambda / 4$, or $3 \lambda / 4$. However, we find, that as these substrings move away from each other, their final shifts will be 0 , or $\lambda / 2$.

\section{Stability}

\section{A. The Stability Equations}

The motion of the test particle around the equilibrium is described by the equations:

$$
\begin{aligned}
y^{\prime \prime}+\left[K_{v}(s)-\frac{4 \lambda_{c}^{3}}{g_{0} R^{2} \lambda^{3}} \eta_{v}\right] y & =0, \\
x^{\prime \prime}+\left[K_{h}(s)-\frac{4 \lambda_{c}^{3}}{g_{0} R^{2} \lambda^{3}} \eta_{h}\right] x & =h \delta, \\
\sigma^{\prime} & =h x-\frac{\delta}{\gamma^{2}}, \\
\delta^{\prime} & =2 \gamma^{2} \frac{4 \lambda_{c}^{3}}{g_{0} R^{2} \lambda^{3}} \eta_{e} \sigma .
\end{aligned}
$$

$\eta_{h, v, e}$ are proportional to the second order expansion of $\Phi_{s c}$ around the equilibrium of the test particle. The space charge force of the crystalline beam perturbs the free-oscillation motion of the test particle. Again, we can use the smooth approximation to find out when the tune crosses the stopband. Only an integral stopband, corresponds to a pitchfork bifurcation. A half integral stopband corresponds to a period doubling, and this violates the confinement conditions, since crystalline beams need to have the same periodicity as the storage ring. The lower limit $\lambda_{l}$ of the ion spacing in the crystalline beam is given by:

$$
\lambda_{l}=\eta_{h, v}^{\frac{1}{3}} \lambda_{c} c_{h, v} .
$$

\section{Results and Discussion}

We investigated a succession of crystaline beams for weak ( $\Delta=0.001)$, and moderate $(\Delta=0.25)$ defocussing. To obtain the envelope we solved Eqs. (8) and (13). The stability followed from Eq. (18). Our results are shown in Figs. 1, and 2. We consider gold ions $(Q=57, A=157)$ in a storage ring with a magnetic rigidity of $1.2 \mathrm{Tm}$, a radius of $6.46 \mathrm{~m}$, a superperiod of 16, and tune $\nu_{h}=4.8 . \nu_{v}=4.8$, for $\Delta=0.001$, and 5.54, for $\Delta=0.25 ; \lambda_{c}=47 \mu \mathrm{m} . \frac{\gamma^{2}}{R^{2}}$ is small, so we have ignored it.

In our analysis, we start with the string. There is no upper limit to $\lambda$; the string is formed even by very dilute ion clouds. In the smooth approximation, the lower limit is determined by the horizontal, and vertical stopband of the storage ring. Using transfer matrices, we determined however, that for most realistic storage rings, the coupling resonance between the longitudinal, and transverse motion in the horizontal plane is quite important.

The focussing in the vertical direction is the largest, and the next stucture is a horizontal zigzag. Using transfer matrices we determined that only extremely smooth storage rings can support horizontal zigzags. Therefore, for storage rings that support higher order crystaline beams, the smooth approximation must be accurate.

At the point of bifurcation, we find that the ion spacing of the zigzag is roughly twice that of the string from which it originates. In addition, to balance the longitudinal forces, the two substrings are shifted by half an ion spacing. This is all in agreement with the bifurcation picture. For large defocussing $(\Delta>0.5)$, the zigzag bifurcates in the plane. For $\Delta<0.5$, the zigzag bifurcates in the vertical direction, and goes over into a helix. Again, the ion spacing roughly doubles at bifurcation. 


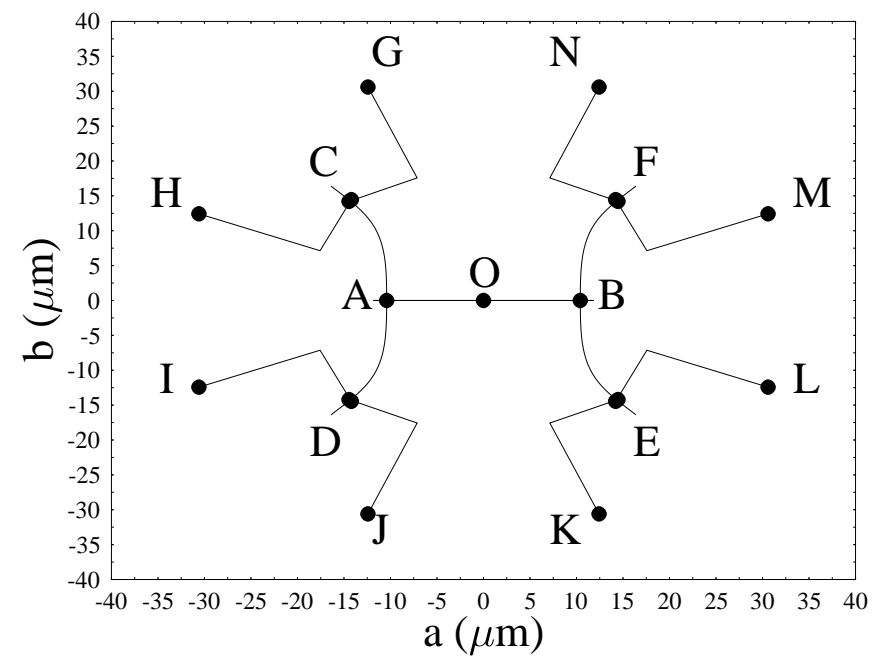

Figure 1. The transition from string (0), to horizontal zigzag (AB), to helix (CDEF), and to shell(16) (GHIKL ...) using the smooth approximation, for $\Delta=0.001$.

To balance the longitudinal forces, the four substrings shift a little bit longitudinally as they move away from each other. This transition region is very small.

Eventually, the helix will become unstable, and its four substrings will each split in two, to form a shell consisting of eight substrings. We call this structure shell(8), to distinguish it from other shells. The ion spacing doubles again, and the substrings shift longitudinally as they move away from each other, in order to maintain longitudinal equilibrium.

The next shell structure, shell(16), is formed when the eight substrings of the shell(8) split. It is unstable right from the start. Therefore, a shell with eight substrings is the largest hollow crystalline beam we obtained.

Putting a string in the middle of the shell(16), along the reference orbit, stabilizes it. This is in agreement with the MD simulations of Wei et al. [3].

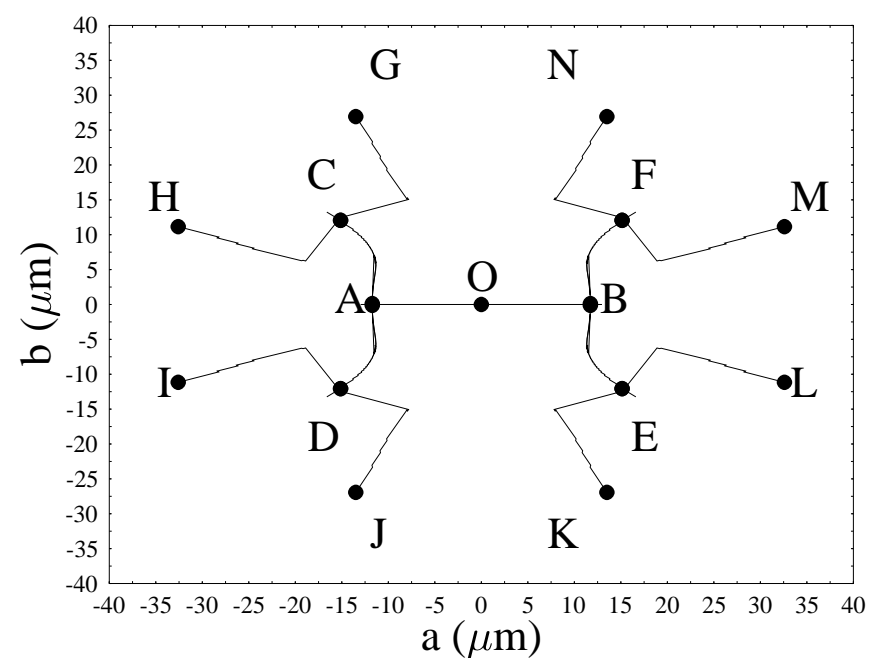

Figure 2. The transition from string (0), to horizontal zigzag (AB), to helix (CDEF), and to shell(16) (GHIKL ...) using the smooth approximation, for $\Delta=0.25$

\section{References}

[1] J.P. Schiffer and A. Rahman, Feasibility of a Crystalline Condensed State in Cooled Ion Beams of a Storage Ring. PHY-5121-ME-88. Feb. 1988, Argonne National Laboratory. R.W. Hasse and J.P. Schiffer, Ann. of Phys. 203, 419 (1990).

[2] H. Walther, Phase Transitions of Laser-Cooled Ions, in Advances in At. Mol. and Opt. Physics, 31, p.137, (1993), and, Atoms in Cavities and Traps, in Advances in At. Mol. and Opt. Physics, 32,p. 379, (1994).

[3] J.Wei, X.P.Li, A.M.Sessler, Crystaline Beam Ground State, BNL-52381, June 1993.

[4] E.D. Courant and H.S. Snyder, Ann. of Phys. 3,1 (1958).

[5] John Guckenheimer and Philip Holmes, Nonlinear Oscillations, Dynamical Systems, and Bifurcations of Vector Fields, Springer-Verlag, New York, 1983.

[6] J.A.G. Roberts and G.R.W. Quispel, Phys. Rep. 216, 63 (1992).

[7] A.F. Haffmans, D. Maletić, and A.G. Ruggiero, Informal Report, BNL-60436, April 1994, Informal Report, BNL60619, April 1994, Informal report, BNL-60743, May 1994, Informal report, BNL-60876, May 1994; Confinement and Stability of Crystalline Beams in Storage Rings, To appear in the "Proceedings of the 6th Workshop on Advanced Accelerator Concepts", June 12-18, 1994, BNL60878, September 1994. 